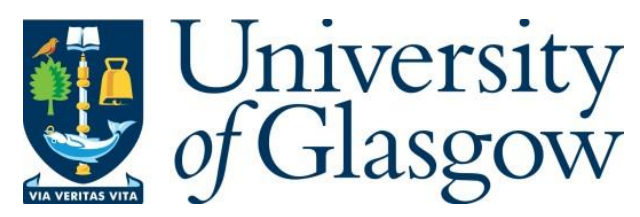

Liang, J. et al. (2017) A Physics-based Investigation of Pt-salt Doped Carbon Nanotubes for Local Interconnects. In: 2017 IEEE International Electron Devices Meeting (IEDM), San Francisco, CA, USA, 02-06 Dec 2017, 35.5.1-35.5.4. ISBN 9781538635599.

There may be differences between this version and the published version. You are advised to consult the publisher's version if you wish to cite from it.

http://eprints.gla.ac.uk/159398/

Deposited on: 20 March 2018

Enlighten - Research publications by members of the University of Glasgow http://eprints.gla.ac.uk 


\title{
A Physics-Based Investigation of Pt-Salt Doped Carbon Nanotubes for Local Interconnects
}

\author{
J. Liang 1 , R. Ramos 2 , J. Dijon², H. Okuno 3 , D. Kalita 3 , D. Renaud ${ }^{2}$, J. Lee ${ }^{4}$, V. P. Georgiev ${ }^{4}$, S. Berrada ${ }^{4}$, T. Sadi ${ }^{4,8}$, \\ A. Asenov ${ }^{4}$, B. Uhlig ${ }^{5}$, K. Lilienthal ${ }^{5}$, A. Dhavamani ${ }^{5}$, F. Könemann ${ }^{6}$, B. Gotsmann ${ }^{6}$, G. Goncalves ${ }^{7}$, B. Chen ${ }^{7}$, \\ K. Teo ${ }^{7}$, R. R. Pandey ${ }^{1}$, and A. Todri-Sanial ${ }^{1}$ \\ ${ }^{1}$ CNRS/LIRMM-University of Montpellier, France; ${ }^{2}$ University Grenoble Alpes/CEA-LITEN, France; \\ ${ }^{3}$ University Grenoble Alpes/CEA-INAC, France; ${ }^{4}$ School of Engineering, University of Glasgow, UK; ${ }^{5}$ Fraunhofer IPMS, \\ Dresden, Germany; ${ }^{6}$ IBM Research Zurich, Switzerland; ${ }^{7}$ Aixtron Ltd., Cambridge, UK; ${ }^{8}$ Aalto University, Finland.
}

\begin{abstract}
We investigate, by combining physical and electrical measurements together with an atomistic-to-circuit modeling approach, the conductance of doped carbon nanotubes (CNTs) and their eligibility as possible candidate for next generation back-end-of-line (BEOL) interconnects. Ab-initio simulations predict a doping-related shift of the Fermi level, which reduces shell chirality variability and improves electrical conductance up to $90 \%$ by converting semiconducting shells to metallic. Circuit-level simulations predict up to $88 \%$ signal delay improvement with doped vs. pristine CNT. Electrical measurements of Pt-salt doped CNTs provide up to $50 \%$ of resistance reduction which is a milestone result for future CNT interconnect technology.
\end{abstract}

\section{INTRODUCTION}

Semiconductor industry requires fast, dense and highbandwidth interconnects to manufacture high-performance systems. In the current technology, interconnects are realized by using copper $(\mathrm{Cu})$ with low-k inter-layer dielectric material using dual damascene process. The geometrical dimensions of the trenches and the material used as a conductor determine the resistivity. As the dimensions of $\mathrm{Cu}$ wire decrease and become comparable or smaller than its electron mean free path (MFP), size effects such as electron scattering at wire surface and grain boundaries, and line edge roughness cause the effective resistivity to increase rapidly [1-2], also shown in Fig. 1 [3]. Increase in resistivity of $\mathrm{Cu}$ lines will inevitably cause performance degradation as time delay is dominated by resistance and capacitance ( $\mathrm{R}$ and $\mathrm{C}$ ).

Atomically thin, ballistic transport, large electron MFP and highly conductive carbon based materials such as carbon nanotubes (CNTs) provide compelling advantages for the next generation of on-chip interconnects [4] CNTs are also envisioned as one of the future materials for advanced technology node based on IRDS report [5]. In this paper we present Pt-salt doped CNTs as a viable solution to enhance CNT conductivity, control chirality variation and improve signal delay as a premise for future local interconnect technology nodes.

\section{Physical Modeling OF PT-SAlt doped CNT}

Doping of CNTs presents an effective solution to overcome variations on random chirality and shell variability in MWCNTs. Doping shifts material Fermi-Level, thus enhancing metallic properties for semiconducting tubes and reducing metallic tubes resistivity by introducing additional electron transport channels.

\section{A. Atomistic model: DFT calculation}

We have performed the Density Functional Theory (DFT) simulations, which is implemented in Atomistix Tool Kit (ATK) [6] to investigate the electrical properties of Pt-salt doped CNTs. The generalized gradient approximation (GGA) is applied for the exchange-correlation energy functional. We have compared the formation energies with various atomic structures as shown in Fig. 2. When $\mathrm{PtCl}_{4}$ is located inside the CNT (Fig. 2 (b)), the structure is most stable. Fig. 3 (a) shows the band structure and density-of-state (DOS) of pristine and doped CNT $(15,0)$. We have found that Pt-salt is a good p-type dopant. Thanks to the Fermi-level $\left(\mathrm{E}_{\mathrm{F}}\right)$ shift, doped CNT has larger DOS and number of conducting channel $(\mathrm{Nc})$ near $\mathrm{E}_{\mathrm{F}}$. The band structure and DOS of the semiconductor CNT $(16,0)$ are shown in Fig. 3 (b). Due to $\mathrm{E}_{\mathrm{F}}$ shift, the semiconductor CNT can have a metallic behavior. The value of $E_{F}$ shift in double walled CNTs is also calculated, and the results are shown in Table 1. To summarize, doping of CNTs reduces the chirality variability of shells by degenerates semiconducting shells and converting them into metallic.

\section{B. Circuit-level electrical $R C$ model}

MWCNT shells have different number of conducting channels due to shell chirality variability. We introduce doping enhancement factor Nc - average number of conducting channels per shell - to represent a control of chirality variability as known that doping will convert semiconducting shells into metallic properties. One-channel conductivity is given by $G_{\text {-channel }}=G_{0} /\left(1+L / L_{M F P}\right) . \quad G_{0}$ is quantum conductance $(\sim 1 / 12.9 \mathrm{k} \Omega))$. $L_{M F P}$ is used for metallic CNT [7]. CNT has quantum $\left(\mathrm{C}_{\mathrm{Q}}\right)$ and electrostatic capacitance $\left(\mathrm{C}_{\mathrm{E}}\right)$ [8]. Quantum capacitance per channel $C_{Q-1}$ channel is given as $96.5 \mathrm{aF} / \mu \mathrm{m}$ [8], doping linearly increases total quantum capacitance, however, $\mathrm{C}_{\mathrm{E}}$ is geometry dependent and does not depend on doping. Electrical modeling is shown in Fig. 4, and total doped MWCNT resistance and capacitance are derived as:

$$
R_{M W}=1 /\left(N c \cdot N s \cdot G_{\text {I-channel }}\right)
$$

$C_{M W}=N c N s C_{Q-1 \_c h a n n e l} \cdot C_{E} /\left(N c N s C_{Q-1 / c h a n n e l}+C_{E}\right) \approx C_{E}$ (2) Number of shells $(N S)$ is derived as diameter-1 based on experimental statistical measurements. Fig. 5 shows the CNT 
conductivity variation for a $7 \mathrm{~nm}$ diameter MWCNT for lengths up to $10 \mu \mathrm{m}$ while varying Nc. The doped CNTs have higher conductance than pristine CNTs and $\mathrm{Cu}$ lines (32, 22 and 14 $\mathrm{nm})$. We notice that conductance of pristine CNTs becomes advantageous to $\mathrm{Cu}$ lines for lengths beyond $3 \mu \mathrm{m}$. With doping, CNTs become advantageous at even shorter lengths, which indicate the suitability of doped CNTs for local short on-chip interconnects where $\mathrm{Cu}$ line performance is significantly degraded due to surface scattering. In Fig. 6, resistance and capacitance change with doping for a $7 \mathrm{~nm}$ diameter MWCNT is shown where up to $90 \%$ reduction is obtained with $\mathrm{Nc}=5$. The resistance reduction proves the decrease of CNT shell chirality variability due to doping. Doping only impacts $\mathrm{C}_{\mathrm{Q}}$, while total CNT capacitance is dominated by $\mathrm{C}_{\mathrm{E}}\left(\mathrm{C}_{\mathrm{MW}}\right.$ increases by $9 \%$ with $\left.\mathrm{Nc}=5\right)$. Hence, overall RC delay computed through Elmore formula (delay: $\tau$ $\sim \mathrm{R}_{\mathrm{MW}} \mathrm{C}_{\mathrm{MW}}$ ) improves with doping due to resistance reduction.

To further analyze the impact of doped CNTs on local interconnect, we compute the delay ratio for MWCNTs diameter 5 to $10 \mathrm{~nm}$, length $10 \mathrm{~nm}$ to $100 \mu \mathrm{m}$ and $\mathrm{Nc} 1$ to 5 (representing light to heavily doped CNTs) shown in Fig. 7. Overall, we observe that doping improves signal delay. As CNT diameter gets large, doping becomes less efficient as already large diameter CNTs have metallic properties. Fig. 8 shows that doped CNT interconnects also reduce delay and diameter variability.

\section{CNT GROWTH AND DOPING PROCESS}

\section{A. Localized CNT growth}

CNT growth is achieved by hot filament assisted catalytic chemical vapor deposition (CVD) using iron catalyst and acetylene carbon precursor at temperature around $600^{\circ} \mathrm{C}$. To ensure controlled placement of CNT, the catalyst is localized at the bottom of via holes prepared by standard nanofabrication technology (Fig. 9). A unique method was developed to grow individual small diameter MWCNT with controlled structure. It relies on the formation of a single catalyst droplet - hence a single CNT - in the via hole during CVD growth. It is achieved by precisely tuning the quantity of catalyst, the nature of the catalyst support and CNT growth parameters. Typically, the growth of individual MWCNT can be achieved on aluminosilicate support with $1 \mathrm{~nm}$ thick iron catalyst if the hole diameter is less than $40 \mathrm{~nm}$ (Fig. 9). Fig. 10 displays TEM images of individual CNTs grown from via holes with a diameter of $30 \pm 10 \mathrm{~nm}$. It also shows the distribution of CNT diameter $(6.5 \mathrm{~nm} \pm 1 \mathrm{~nm})$ and number of shells (mostly 4 and 5 walls). CNTs grown on $300 \mathrm{~mm}$ wafers are processed using AIXTRON BM 300T system. The CNT growth occurs in a cold wall reactor using a vertical gas flow distributed via a showerhead. This configuration allows a fast scaling of the process conditions from the coupon size to the $300 \mathrm{~mm}$ wafer scale.

\section{B. $\mathrm{PtCl}_{4}$ internal doping process}

Structural opening of the tip of CNT is necessary for effective internal doping of CNTs. It allows for the dopants to enter the inner tubes of the CNT and remain confined. We find that CNTs treated with oxygen plasma under mild conditions ensure high CNT filling rate of $30 \%$. This filling rate is not absolute but could vary with $\mathrm{PtCl}_{4}$ concentration and density of CNTs. Figure 11 (a) shows HAADF-TEM image of the CNT opened with oxygen plasma process and doped with Pt$\mathrm{Cl}$ network of dopants. The three different CNTs are highlighted with doped doted purple lines. The $\mathrm{Pt}$ and $\mathrm{Cl}$ elements within the doped-CNT were verified using EnergyDispersive X-ray spectroscopy (EDX) mapping shown in Fig. 11 (c). The difference in concentration between neighboring CNTs is due to partial amorphization induced by plasma treatment. Based on these results, a dedicated process flow (shown in Figs. 12-13) for contact fabrication and internal doping of integrated CNT was developed.

\section{ELECTRICAL MEASUREMENTS}

A set of individual MWCNT interconnect fabricated in parallel on the same sample was electrically characterized. The CNTs were grown from $30 \pm 10 \mathrm{~nm}$ diameter via holes and side-contacted with Pd according to the process flow in Fig. 12. Two-point-probe current-voltage characteristics were systematically recorded to extract the electrical resistance (CNT resistance + CNT/Pd side contact resistance). Fig.14 (a) displays the measured resistance as a function of the CNT length. It is observed that resistance generally increases with CNT length in the $1-10 \mu \mathrm{m}$ range, which is expected for diffusive transport in CVD-grown, hence, defective CNTs. However, a large scatter in the data is observed, thus revealing variability in MWCNT conductivity and contact resistance. The origin of the variability may be the variability in the diameter, number of shells of each CNT (cf Fig. 10), the random repartition of metallic versus semiconducting shells, the presence of defects, or variability in the contact fabrication technology. Assuming the measured resistance is dominated by the resistivity of MWCNT, linear resistance is comprised between 55 and $235 \mathrm{k} \Omega / \mu \mathrm{m}$ ( $90 \%$ envelope). Preliminary electrical results of doped CNTs by $\mathrm{PtCl}_{4}$ were obtained by using external doping of side-contacted single CNT interconnects. Fig.14 (b) displays the measured linear resistance (resistance divided by CNT length) for the same set of CNTs before and after external doping. It is observed that, on average, the resistance of externally doped MWCNTs is reduced by a factor of two. The scatter of data is also reduced which suggests variability improves with doping.

\section{CONCLUSION}

In this paper, we investigate Pt salt doped CNT for BEOL interconnects. By combining atomistic to circuit-level simulation and measurement results, we explain the impact of doping on enhancing MWCNT metallic properties. Both experimental data and simulations show that the $\mathrm{PtCl}_{4}$ solution represents an efficient doping strategy to improve MWCNT performance and reduce the intrinsic statistical variation associated with chirality and diameter fluctuations. This represents a milestone result, which could enable the success of MWCNTs as candidate for future technology nodes interconnects. 


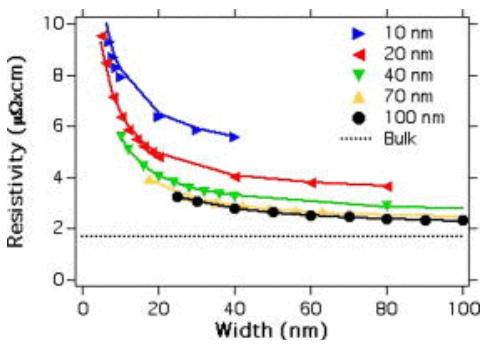

Fig. 1. $\mathrm{Cu}$ resistivity vs. $\mathrm{Cu}$ line width for different line heights.

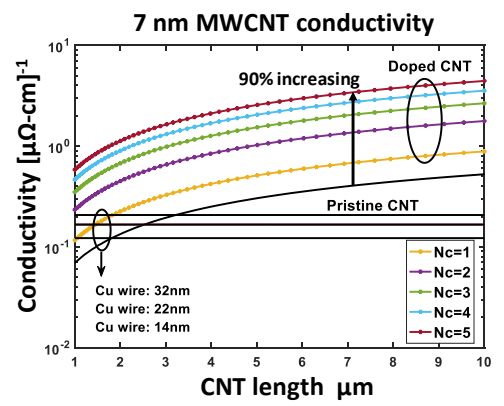

Fig. 5. $7 \mathrm{~nm}$ diameter MWCNT line conductivity with different doping concentration (increase of $\mathrm{Nc}$ indicates more doping concentration).

Fig. 6. RC delay calculation for $7 \mathrm{~nm}$ doped MWCNT. Nc reduces $87.5 \%$ of RC delay with R decreases by $88.8 \%$ and $\mathrm{C}$ increases by $9 \%$.

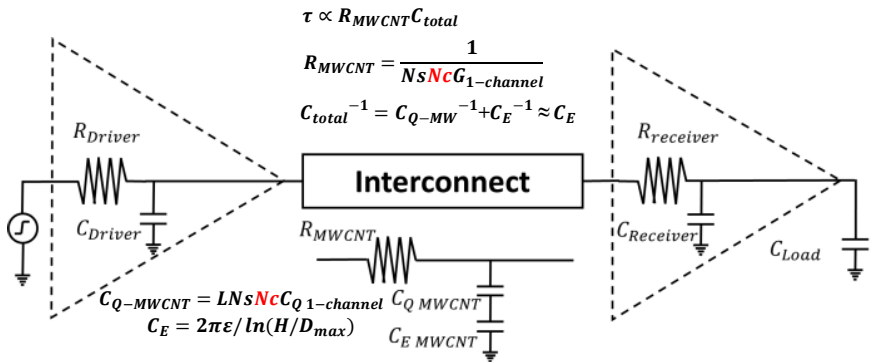

Fig. 4. Electrical model of doped MWCNT as interconnect. Analytical formulas for R and $\mathrm{C}$ of doped MWCNT.

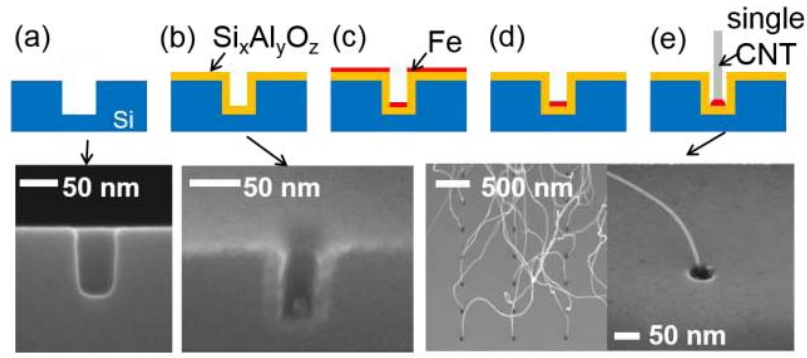

Fig. 9. Localized growth of a single MWCNT from a via hole. (a) Via hole prepared in silicon substrate by E-beam lithography and plasma etching. (b) Conformal deposition of aluminosilicate by atomic layer deposition to trim the hole diameter. (c) Directional E-beam evaporation of $1 \mathrm{~nm}$ Fe catalyst at normal incidence. (d) Ion beam etching of top surface catalyst at grazing incidence. (e) CNT growth by hot filament CVD.
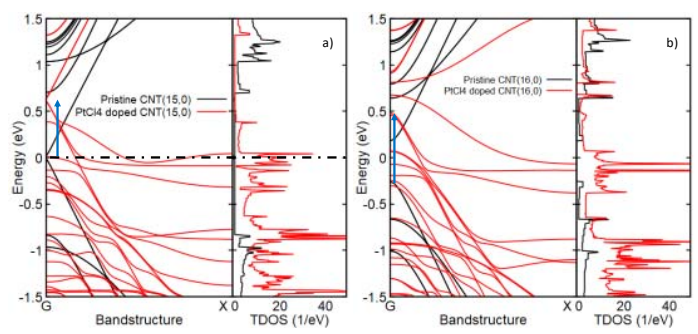

Fig. 3. Band structure and total DOS of pristine and $\mathrm{PtCl}_{4}$ doped (a) CNT $(15,0)$ (See Fig 2. b)). Fermilevel $\left(\mathrm{E}_{\mathrm{F}}\right)$ is set to $0.0 \mathrm{eV}$. Due to the dopant, $\mathrm{E}_{\mathrm{F}}$ is shifted by $-0.6 \mathrm{eV}$, and DOS near $\mathrm{E}_{\mathrm{F}}$ has increased. (b) $\mathrm{CNT}(16,0) . \quad \mathrm{E}_{\mathrm{F}}$ shifts by dopants, semiconductor CNT $(16,0)$ has become a metal.

Table. 1. Summary of $E_{F}$ shift of DWCNTs. 'M' and ' $\mathrm{S}$ ' indicate the metallic and semiconductor of each shell. $E_{F}$ shift is calculated based on the valence band edge.

\begin{tabular}{|c|c|c|}
\hline & $\begin{array}{c}\text { Fermi-level } \\
\text { shift } \\
\text { (inner shell) }\end{array}$ & $\begin{array}{c}\text { Fermi-level } \\
\text { shift } \\
\text { (outer shell) }\end{array}$ \\
\hline CNT $(15,0)(24,0)$ M,M & $\mathbf{0 . 5 0 9} \mathbf{~ e V}$ & $\mathbf{0 . 4 4 4} \mathbf{~ e V}$ \\
\hline CNT $(15,0)(25,0)$ M,S & $\mathbf{0 . 5 5 4}$ eV & $\mathbf{0 . 5 0 2} \mathbf{~ e V}$ \\
\hline CNT $(14,0)(24,0)$ S,M & $\mathbf{0 . 5 8 6}$ eV & $\mathbf{0 . 5 0 3} \mathbf{~ e V}$ \\
\hline CNT $(14,0)(23,0)$ S,S & $\mathbf{0 . 5 5 6}$ eV & $\mathbf{0 . 4 2 8}$ eV \\
\hline
\end{tabular}

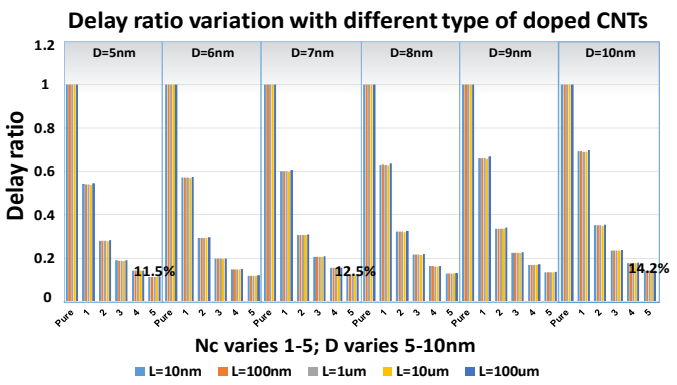

Fig. 7. Delay ratio after/before doping. Nc varies from 1 to 5 , MWCNTs with $\mathrm{D}=5-10 \mathrm{~nm}$ and $\mathrm{L}=10 \mathrm{~nm}-100 \mu \mathrm{m}$. Doping reduces delay ratio of $88.5 \%$ and $85.8 \%$ for $\mathrm{D}=5 \mathrm{~nm}$ and $10 \mathrm{~nm}$. With larger diameter, doping gets less efficient on delay ratio, as large diameter MWCNT already has metallic properties.

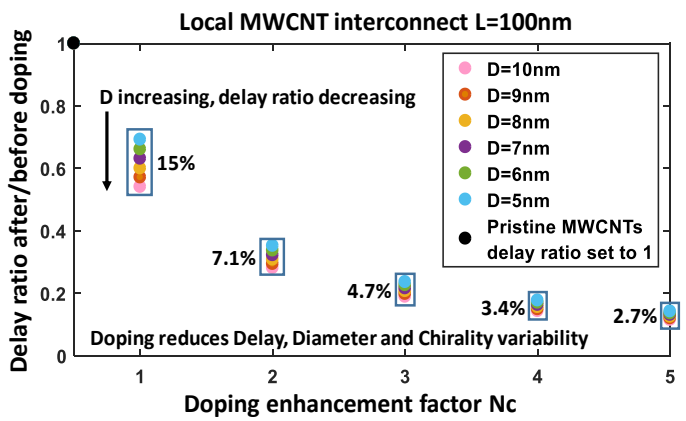

Fig. 8. Diameter variability is reduced by doping from $15 \%$ to $2.7 \%$ with respect to $\mathrm{Nc}$ varies from 1 to 5 . 


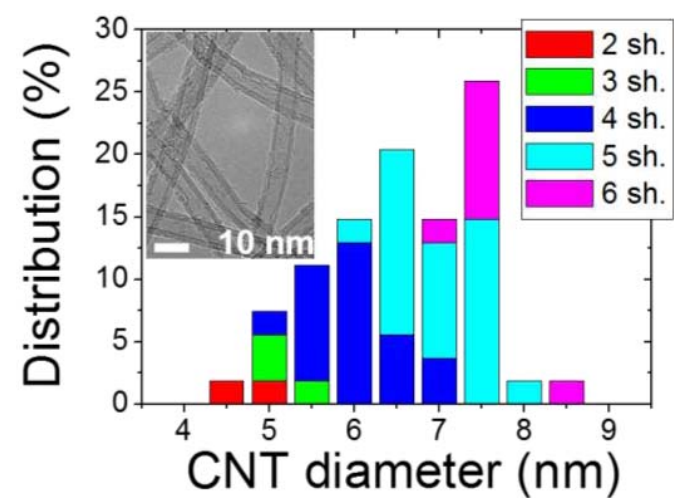

Fig.10. Statistical distribution of the diameter and number of shells of individually grown CNTs from $30 \pm 10 \mathrm{~nm}$ diameter via hole with representative TEM image (inset). Median CNT diameter is $6.5 \pm 1 \mathrm{~nm}$ with 4-5 shells.
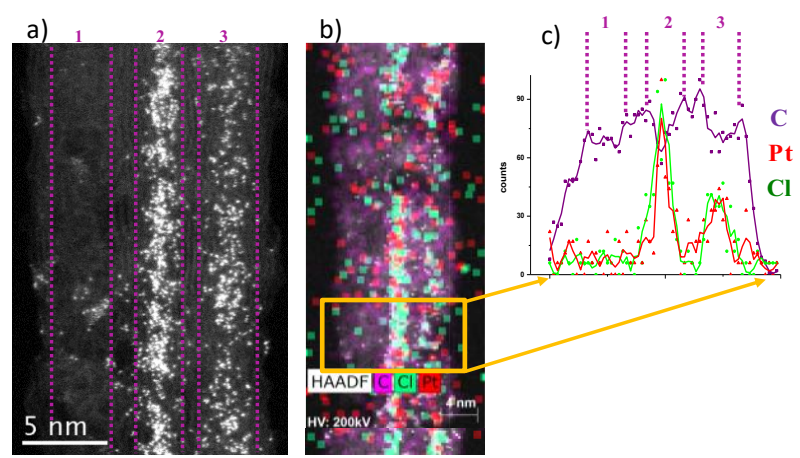

Fig. 11. (a) HAADF-TEM image of CNTs with different concentration of dopant (purple dotted lines shows the different CNTs). (b) EDX mapping of Pt-Cl doped CNTs. (c) Line scan of EDX mapping across doped CNT. Purple, red, green color represents Carbon, Platinum and Chlorine respectively.

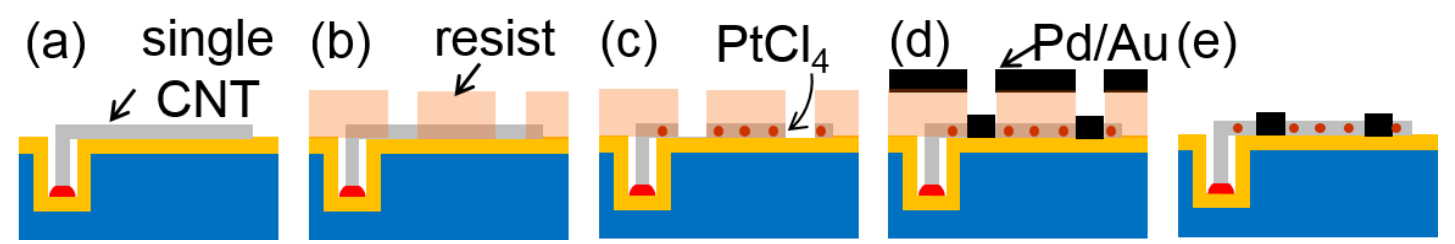

Fig. 12. Process flow for internally-doped end-contacted single CNT interconnect line. (a) Single CNT grown from via hole and flipped down on the surface. (b) Photolithography to pattern the contact pads. (c) Plasma etching of CNT followed by dipping in $\mathrm{PtCl}_{4}$ solution to introduce dopant inside the CNT. (d) Physical vapor deposition of Pd/Au contact metal stack. (e) Contact pad fabrication by lift-off. The same process flow is used to fabricate side-contacted CNT interconnect by suppressing step (c). External doping of CNT is achieved by dipping the sample after step (e) in the dopant solution.

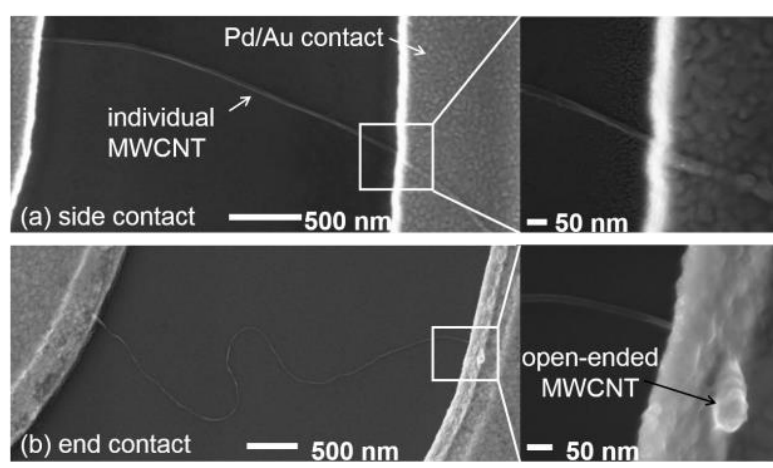

Fig. 13. SEM images of electrically connected individual MWCNT with (a) side and (b) end contacts made out of Pd (40nm) and $\mathrm{Au}(150 \mathrm{~nm})$.

\section{ACKNOWLEDGMENT}

Authors would like to acknowledge H2020 CONNECT European project. This project has received funding from the European Union's Horizon 2020 research and innovation program under grant agreement No 688612. (http://www.connect-h2020.eu/)

R.R. and J.D. would like to acknowledge Intel Oregon for financial support on CNT TEM characterization
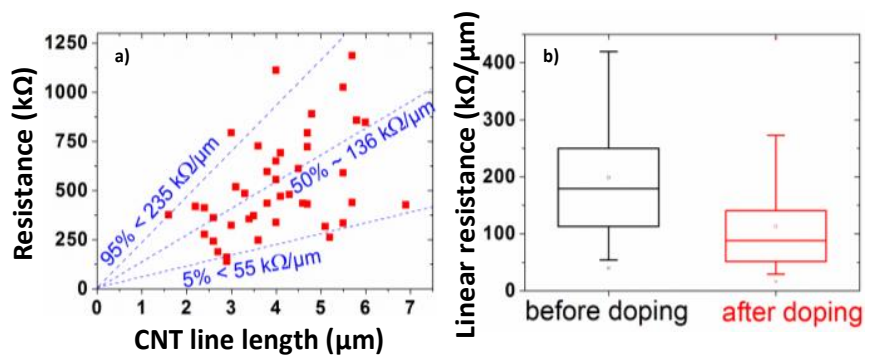

Fig. 14. (a) Two points probe resistance measurement for a collection of individual CNTs. (b) 50\% improvement of linear resistance with external $\mathrm{PtCl}_{4}$ doping.

\section{REFERENCES}

[1] Im, Sungjun, et al., IEEE TED 52.12 (2005): 2710-2719. [2] Steinhögl, Werner, et al. Journal of Applied Physics. 97.2 (2005): 023706. [3] Graham, Rebekah L., et al. Applied Physics Letters 96.4 (2010): 042116. [4] A. TodriSanial, J. Dijon, et al. "Carbon nanotube interconnects: process, design and application," Springer 2016; [5] IRDS 2017 http://irds.ieee.org/reports. [6] Atomistix Tool Kit (2016.4), QuantumWise A/S. [7] Naeemi, Azad, and James D. Meindl. IEEE Electron Device Letters 27.5 (2006): 338-340. [8] Li, Hong, et al., IEEE TED 55.6 (2008): 1328-1337. 